\section{(6) OPEN ACCESS}

'Division of Brain Sciences, Imperial College London, London, UK

${ }^{2}$ Centre for Cognition and Neuroimaging, Department of Psychology, Brunel University, Greater London, UK

Correspondence to Dr Paresh Malhotra, Division of Brain Sciences, Imperial College London, 10 E Charing Cross Campus, London W6 8RP, UK. p.malhotra@imperial.ac.uk

Received 7 May 2012 Revised 9 August 2012 Accepted 22 August 2012 Published Online First 15 October 2012

\title{
Reward modulates spatial neglect
}

\author{
Paresh A Malhotra, ${ }^{1}$ David Soto, ${ }^{1}$ Korina Li, ${ }^{1}$ Charlotte Russell ${ }^{2}$
}

\begin{abstract}
Background Reward has been shown to affect attention in healthy individuals, but there have been no studies addressing whether reward influences attentional impairments in patients with focal brain damage. Methods Using two novel variants of a widely-used clinical cancellation task, we assessed whether reward modulated impaired attention in 10 individuals with left neglect secondary to right hemisphere stroke.

Results Reward exposure significantly reduced neglect, as measured by total targets found, left-sided targets found and centre of cancellation, across the patient group. Lesion analysis showed that lack of response to reward was associated with damage to the ipsilateral striatum.

Conclusions This is the first experimental evidence that reward can modulate attentional impairments following brain damage. These results have significant implications for the development of behavioural and pharmacological therapies for patients with attentional disorders.
\end{abstract}

\section{INTRODUCTION}

Despite the disabling consequences of hemispatial neglect there are presently no widely recognised effective treatments, although evidence suggests that some therapies, including dopaminergic stimulation and, in particular prism adaptation, may be of benefit in selected patients. ${ }^{1-3}$ The wave of recent research demonstrating that reward influences attention in healthy humans raises the critical question of whether reward can improve attention in neglect. ${ }^{4}$ Studies with healthy participants have suggested that pairing monetary reward with specific stimuli results in preferential allocation of attention to these stimuli, whether this enhances or worsens task performance. ${ }^{4} 5$ Although such data show that reward exerts a powerful influence on attention and clinical observation has suggested that this influence may extend to patients, ${ }^{6}$ no studies to-date have addressed whether reward might influence attentional impairments following brain damage. Here, using a novel variation of a common diagnostic cancellation test, we examine the effects of reward on neglect.

\section{METHODS \\ Patients}

All patients were recruited from Imperial College Healthcare NHS Trust, London (see table 1 for details) and had left neglect secondary to right hemisphere stroke. All patients were right-handed and none of the patients had previously suffered from neurological disease. Because neglect tends to be most severe in the early period following stroke and early interventions may lead to long-term benefit, ${ }^{7}$ we included acute and chronic patients.

\section{Experimental tasks}

To examine for the effects of reward, we employed two adapted versions of a standard cancellation task (figure 1). The task was administered in a standard clinical fashion. That is, the experimenter sat opposite the patients, who were asked to circle all the targets that they could find on each array, and to inform the experimenter when they could not find any more. Target stimuli (figure 1B) were $£ 1.00$ coins in the Reward (R) task. ${ }^{8}$ As neglect can improve or worsen spontaneously in the acute and chronic stages, ${ }^{9}$ we included a baseline No-Reward (NR) control task where targets were brass buttons.

Prior to testing, patients were informed that they would receive a reward for each target that they found on the $\mathrm{R}$ task only. After completing both tasks in Session 1 all patients received vouchers worth $£ 15$ (awarding identical amounts was requested by the Ethics committee) but crucially they were informed that this was based directly on their performance on the $\mathrm{R}$ task.

As there was no preceding training session and patients received no online feedback about accrued reward gain (which has been shown to affect search $^{4}$ ), patients were reassessed in a second session on a separate day to examine for the effects of reward exposure. At the start of Session 2, they were again informed that they would receive a reward based on the $\mathrm{R}$ task alone. At the end participants were again given vouchers worth $£ 15$. R and NR tasks were run once in each session; task order was randomised and counterbalanced across patients and sessions.

\section{Lesion mapping}

Lesions were imaged by MRI (except Patient 3 (CT)) and plotted using a tablet (Wacom Intuos) and MRIcron (http://www.mccauslandcenter.sc. edu/mricro/index.html).

\section{RESULTS}

Total cancellation performance

Figure $1 \mathrm{C}$ shows the total number of targets found in each condition and session across the search array. Data were not normally distributed, so conservative non-parametric statistics were used. A Friedman's test revealed an overall significant difference between tasks and sessions $\left(X^{2}(3)=10.96\right.$, $p<0.05)$. Wilcoxon tests investigated the source of this difference. Target detection in Session 1 did not significantly differ between $\mathrm{R}$ and NR tasks (Wilcoxon Test, $\mathrm{Z}=-0.984$, n.s.), confirming that 
Table 1 Patient demographics and neglect scores

\begin{tabular}{lcclll}
\hline Patient & & $\begin{array}{l}\text { Time } \\
\text { since } \\
\text { stroke } \\
\text { (days) }\end{array}$ & $\begin{array}{l}\text { Star } \\
\text { cancellation } \\
\text { (L/R) }\end{array}$ & $\begin{array}{l}\text { Line } \\
\text { bisection } \\
\text { (mm) }\end{array}$ & $\begin{array}{l}\text { Time } \\
\text { between } \\
\text { sessions } \\
\text { (days) }\end{array}$ \\
\hline 1 & 67 & 97 & $0 / 7$ & 22 & 22 \\
2 & 60 & 436 & $0 / 16$ & 6 & 7 \\
3 & 42 & 321 & $11 / 27$ & 10 & 8 \\
4 & 70 & 10 & $17 / 21$ & 0 & 1 \\
5 & 66 & 62 & $0 / 12$ & 10 & 3 \\
6 & 61 & 2 & $0 / 5$ & 90 & 12 \\
7 & 46 & 1520 & $15 / 27$ & -3 & 3 \\
8 & 69 & 154 & $0 / 14$ & 0 & 1 \\
9 & 56 & 441 & $0 / 11$ & 16 & 7 \\
10 & 68 & 6 & $0 / 13$ & 84 & 5 \\
\hline
\end{tabular}

Star cancellation: Number of targets found on each side of the midline (out of a total of 27 on each side). For the current experiment patients were defined as having neglect if they cancelled $>2$ targets more on the right than the left side of the behavioural inattention test cancellation array. Line Bisection: Mean deviation ( $+\mathrm{ve}=$ Rightward) on attempted bisection of three separate $18 \mathrm{~cm}$ centrally located horizontal lines. No patients were included on the basis of line bisection deficits alone. Screening tests, including cancellation and line bisection tasks, were repeated immediately prior to participation to confirm neglect. All participants gave consent according to the Declaration of Helsinki and the study was approved by the National Research Ethics Service.

there was no intrinsic difference in difficulty or target salience between the two conditions. Critically, in Session 2 target detection in the $\mathrm{R}$ task significantly improved (mean increase of $29 \%$ ) compared to Session $1 \quad(Z=-2.148, p<0.05)$. Improvement across sessions did not occur in the NR condition $(Z=-1.176$, n.s. $)$.

\section{Contralesional cancellation performance}

In patients with neglect a key comparison is the examination of target detection on the contralesional side (figure 1D). Friedman's test revealed an overall significant difference between the two tasks across the two sessions $\left(X^{2}(3)=12.44\right.$, $p<0.01)$. As above, contralesional detection was equivalent for $\mathrm{R}$ and NR tasks in Session 1 ( $z=-1.13$, n.s.). However, patients were significantly better at detecting left-sided targets in the $\mathrm{R}$ compared to NR task in Session $2(z=-2.12, p<0.05)$. Further, they were able to detect significantly more stimuli (mean increase of $84 \%)$ in the second session of the $R$ task $(z=-2.20$, $p<0.05)$ but showed no improvement across sessions in the NR condition ( $z=-1.80$, n.s.).

\section{Centre of cancellation}

To confirm this reward-associated reduction of neglect, we compared the 'Centre of Cancellation'10 (a measure of lateralised impairment which effectively identifies the centre of the 'attentional field ${ }^{11}$ ) across tasks and sessions. A Friedman's test on Centre of Cancellation revealed a significant difference between tasks and sessions $\left(X^{2}(3)=10.39, p<0.05\right)$, with Wilcoxon tests confirming a significant leftward shift in the $R$ task $(Z=-2.70$, $p<0.01$ ) from Session 1 to Session 2. Again, there was no difference between $R$ and NR tasks in Session 1 ( $Z=-0.56$, n.s.), but a significant difference between the tasks in Session 2 $(Z=-2.29, p<0.05)$.

\section{Correlations}

Correlations (Kendall's Tau) were carried out for the effect of reward (as measured by the change in successful cancellation across the array for the $\mathrm{R}$ task between Session 1 and
Session 2) and time since stroke, time between sessions, neglect severity, age and lesion volume. These were all non-significant.

\section{Individual performance and anatomy}

Figure 1E shows response to reward for each individual by plotting the difference between tasks across the two sessions. Any change in the difference between tasks across sessions should reveal the effect of reward exposure, as change secondary to fluctuation in neglect severity (ie, spontaneous recovery and deterioration $^{9}$ ) affects both conditions. Therefore any difference between the two tasks across sessions is related to the association of the $\mathrm{R}$ task with monetary incentive. Using this data, individuals were classified as responders on the basis that the difference in their performance between the $\mathrm{R}$ and NR conditions in Session 2 was greater than the upper 95\% CI for the difference between the two conditions in Session 1. Eight individuals fit this criterion, but Patients 2 and 9 showed no evidence of response as they did not show a relative performance increase across sessions in the R compared to the NR task.

We carried out a subtraction analysis to investigate which brain areas were damaged in these two individuals, but were intact in the other patients (figure $1 \mathrm{~F}$ ). The critical region was centred around the right striatum, affecting the putamen (MNI coordinates $26,10,-6 ; 18,8,13)$ and right caudate $(11,10,13$; $14,9,16)$, as well as the pallidum $(22,5,3)$ and extending inferiorly into frontal cortex $(33,34,-6)$.

\section{DISCUSSION}

Here we present the first empirical evidence that reward can modulate attention in neglect. On an adapted clinical test, reward exposure led to patients finding targets for which they were previously unaware, and for which, in the NR condition, they remained unaware. There are a number of potential explanations for the influence of reward on neglect as demonstrated here. One putative mechanism could be through heightened arousal secondary to reward administration. Similar stimuli have been shown to be associated with changes in galvanic skin response $e^{8}$ and increased arousal can enhance spatial awareness in neglect. ${ }^{12}$ Thus reward could lead to increased arousal in the $\mathrm{R}$ condition, resulting in improved performance. This explanation would be compatible with increased motivation levels and attentional effort ${ }^{13}$ during the $\mathrm{R}$ condition after the receipt of incentive.

Another potential explanation relates to increased target salience, a factor which influences search in neglect. ${ }^{14}$ Following incentive gain and performance feedback, the relative salience of the pound targets may be modulated, enabling patients to find more leftward targets and shifting their centre of cancellation towards neglected space. Therefore, reward's effects here might be mediated via arousal, salience or a combination of these mechanisms.

We saw no consequence of reward in Session 1, even though patients had been prospectively informed that they would receive remuneration for their performance on the $R$ task. However, receipt of incentive, and explicit feedback on performance and its relationship to accrued reward, after this session led to improved cancellation. This is in keeping with previous studies of reward's effects on attention, where healthy participants either had training sessions, providing sufficient time to associate stimuli with reward, and/or received online feedback during task performance. ${ }^{4}$

Functional imaging studies have suggested that a number of regions, including posterior cingulate gyrus, striatum and orbitofrontal cortex, are involved in reward's effects on attention. ${ }^{15}$ 
A

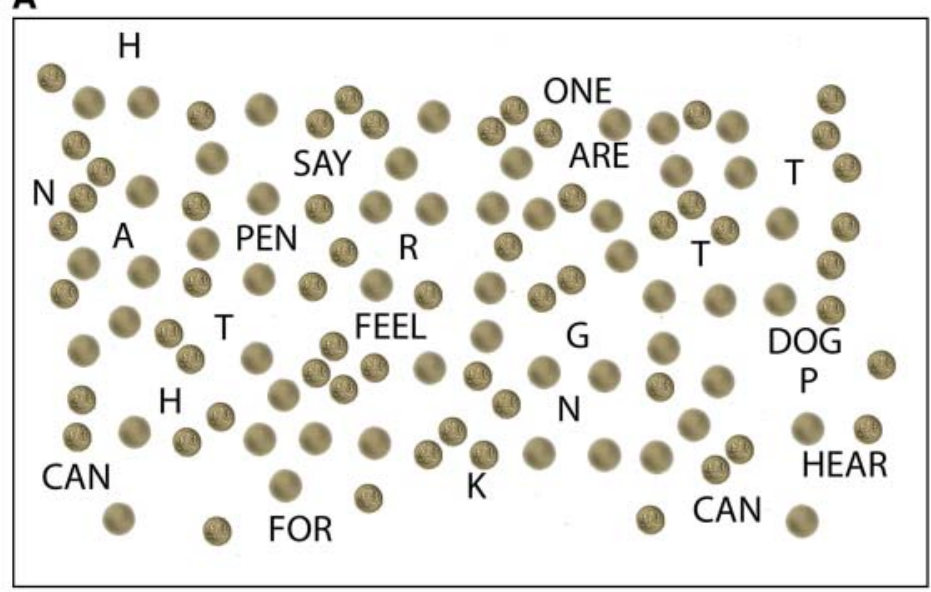

B

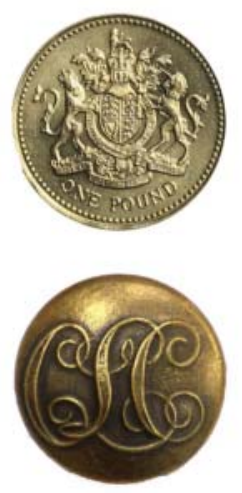

C

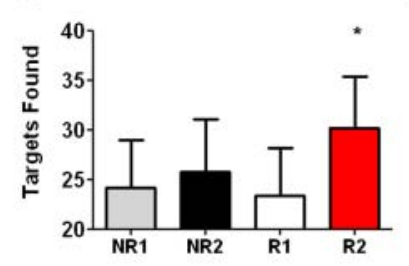

D

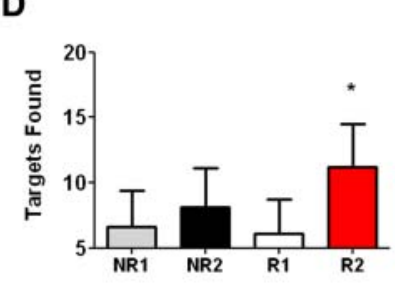

E

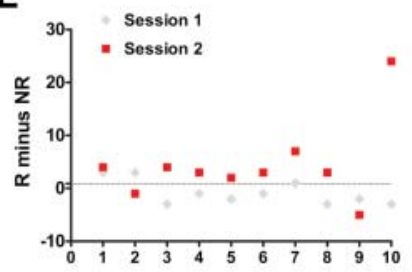

$\mathbf{F}$

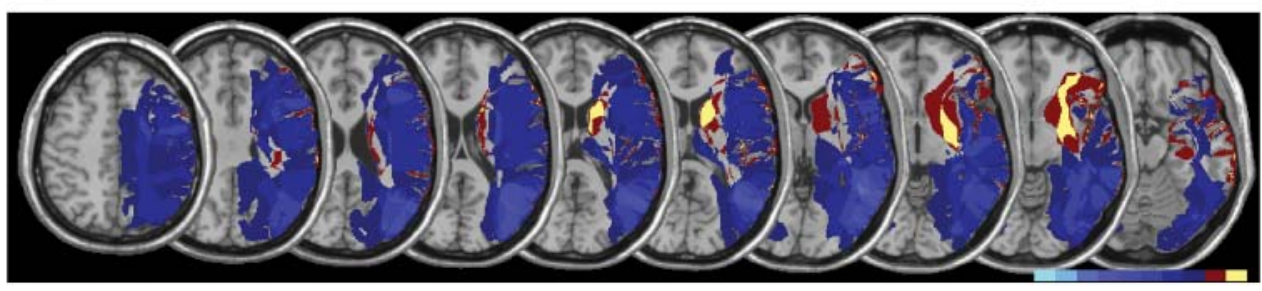

Figure 1 (A) Search array for reward (R) condition. The search array $(420 \mathrm{~mm}$ by $297 \mathrm{~mm}$ ) was a modified variant of the Behavioural Inattention Test (BIT) star cancellation (Bury St. Edmunds: Thames Valley, 1987). There were 54 targets (27 each side of midline) amongst 52 matched distractors; targets and distractors were $12 \mathrm{~mm}$ (diameter). The $\mathrm{R}$ condition employed images of pound coins as rewarding target stimuli. For each session, the search array was placed centrally, and participants were asked to mark every target and inform the experimenter when they could not find any more. Separate matched distractors were produced for each task by using a Gaussian blur to merge the features of each target until they were no longer distinguishable but remained identical in overall distribution of hue and luminance. In addition to the matched distractors, each array contained (as in the original BIT star cancellation) a number of individual letters and words. Each array was constructed using Adobe Photoshop CS3 (Adobe Systems Incorporated). (B) Targets employed in no-reward (NR) and R conditions. The R condition employed images of pound coins as rewarding target stimuli and the NR condition used images of brass buttons as targets that were not explicitly associated with reward. Prior to inclusion, patients were assessed to ensure that they could differentiate between the target and distractor stimuli on both the R and NR task variants. Individuals who could not reliably do this were excluded. (C) Total number of targets found across entire search array on each condition in both sessions. Patients found significantly more targets (mean increase of 6.8 targets) in the second R condition (R2) than in the first (R1). There was no significant difference between the NR condition in Session 2 (NR2) and in Session 1 (NR1). Error Bars: \pm 1 SEM. The maximum number of possible targets is 54 . (D) Number of targets found on the left side in each condition for both sessions. Performance was significantly higher on the neglected side in the second R condition (R2) (mean number of targets found=11.2) than in the first (R1) (mean number of targets found=6.1). Performance was also significantly better in the $R$ condition after reward exposure (R2) compared to the NR condition in the same session (NR2). Error Bars: \pm 1 SEM. The maximum number of possible targets is 27 . (E) Effects of reward exposure at an individual level. Individual patients are represented on the $x$-axis. Grey Diamonds represent the difference between the number of targets found in the R and NR conditions in Session 1 and Red Squares represent the difference between the R and NR conditions in Session 2. The dotted line represents the upper $95 \% \mathrm{Cl}$ from the difference in performance between the R and NR in Session 1. Using this value to separate responders from non-responders, Patients 2 and 9 show no evidence of any response to reward across sessions. Note that, although patient 10 manifests the largest response to reward, the use of non-parametric ranking-based statistics minimises the possibility that one individual's results might be driving the overall significant effects. (F) Lesion Subtraction. Lesions were plotted onto a T1 template consisting of 12 axial slices (MRIcron). The subtraction identifies the brain areas that were damaged in patients who did not show a response to reward $(\mathrm{N}=2)$ and were intact in those that individuals that did $(\mathrm{N}=8)$. The critical region, displayed in bright yellow, was centred around the right striatum (MNI coordinates 11, 10, 13).

Although the patient group was not large enough for voxelbased lesion symptom mapping, our lesion subtraction showed that the principal area damaged in the two non-responding patients but intact in the others, was the striatum. From this preliminary analysis, involvement of ipsilateral striatum seems sufficient to disrupt the effects of reward on neglect, which is in keeping with previous animal work. ${ }^{16}$ Given the key role of dopamine in reward processing and striatal function, it is likely 
that dopaminergic mechanisms are critical for the rewardattention interaction, and dopaminergic agonists have previously been trialled for neglect with varying results. ${ }^{1}{ }^{3} 17$ Previous work has suggested the contribution of a motivational component to neglect, ${ }^{6} 18$ and individual response to motivational influences in the form of rewards may be a predictor of the efficacy of dopamine therapy. Following this new evidence that reward can modulate attentional impairments, further research should enhance understanding of the relationship between reward-based motivation, cognitive deficits and pharmacological interventions.

Acknowledgements The authors would like to thank Jon Driver.

Contributors PM: original conceptualisation of the study, data collection and analysis, drafting the manuscript; DS: original conceptualisation of the study, data analysis, drafting the manuscript; KL: data collection and analysis, drafting the manuscript; CR: original conceptualisation, data analysis, drafting the manuscript.

Funding PM is supported by a HEFCE Clinical Senior Lectureship Award and this research was funded by grants from the UK Academy of Medical Sciences/Wellcome Trust and the NIHR Biomedical Research Centre at Imperial College London. DS is supported by a grant from the UK Medical Research Council (89631). CR is supported by a Brunel Research Initiative Award (BRIEF) and a scientific bursary from the Bial foundation, Portugal.

Competing interests None.

Ethics approval National Research Ethics Service (UK)

Provenance and peer review Not commissioned; externally peer reviewed.

Open Access This is an Open Access article distributed in accordance with the Creative Commons Attribution Non Commercial (CC BY-NC 3.0) license, which permits others to distribute, remix, adapt, build upon this work non-commercially, and license their derivative works on different terms, provided the original work is properly cited and the use is non-commercial. See: http://creativecommons.org/licenses/by-nc/3.0/

\section{REFERENCES}

1. Fleet WS, Valenstein E, Watson RT, et al. Dopamine agonist therapy for neglect in humans. Neurology 1987;37:1765-71.
2. Rossetti Y, Rode G, Pisella L, et al. Prism adaptation to a rightward optical deviation rehabilitates left hemispatial neglect. Nature 1998;395:166-9.

3. Geminiani G, Bottini G, Sterzi R. Dopaminergic stimulation in unilateral neglect. J Neurol Neurosurg Psychiatry 1998;65:344-7.

4. Kristjansson A, Sigurjonsdottir 0, Driver J. Fortune and reversals of fortune in visual search: reward contingencies for pop-out targets affect search efficiency and target repetition effects. Atten Percept Psychophys 2010;72:1229-36.

5. Anderson BA, Laurent PA, Yantis S. Value-driven attentional capture. Proc Natl Acad Sci USA 2011;108:10367-71.

6. Mesulam MM. Principles of behavioral neurology. Philadelphia: F.A. Davis, 1985.

7. Ringman JM, Saver JL, Woolson RF, et al. Frequency, risk factors, anatomy, and course of unilateral neglect in an acute stroke cohort. Neurology 2004;63: 468-74.

8. Pessiglione M, Schmidt L, Draganski B, et al. How the brain translates money into force: a neuroimaging study of subliminal motivation. Science 2007;316:904-6.

9. Farne A, Buxbaum LJ, Ferraro $\mathrm{M}$, et al. Patterns of spontaneous recovery of neglect and associated disorders in acute right brain-damaged patients. J Neurol Neurosurg Psychiatry 2004; 75:1401-10.

10. Rorden C, Karnath HO. A simple measure of neglect severity. Neuropsychologia 2010;48:2758-63.

11. Gainotti G, Perri R, Cappa A. Left hand movements and right hemisphere activation in unilateral spatial neglect: a test of the interhemispheric imbalance hypothesis. Neuropsychologia 2002;40:1350-5.

12. Robertson IH, Mattingley JB, Rorden C, et al. Phasic alerting of neglect patients overcomes their spatial deficit in visual awareness. Nature 1998;395:169-72.

13. Hubner $\mathbf{R}$, Schlosser J. Monetary reward increases attentional effort in the flanker task. Psychon Bull Rev 2010;17:821-6.

14. Bays PM, Singh-Curry V, Gorgoraptis N, et al. Integration of goal- and stimulus-related visual signals revealed by damage to human parietal cortex. J Neurosci 2010;30:5968-78.

15. Small DM, Gitelman D, Simmons K, et al. Monetary incentives enhance processing in brain regions mediating top-down control of attention. Cereb Cortex 2005;15:1855-65.

16. Christakou A, Robbins TW, Everitt BJ. Prolonged neglect following unilateral disruption of a prefrontal cortical-dorsal striatal system. Eur J Neurosci 2005;21:782-92.

17. Grujic Z, Mapstone M, Gitelman DR, et al. Dopamine agonists reorient visual exploration away from the neglected hemispace. Neurology 1998;51:1395-8.

18. Ishiai S, Sugishita M, Odajima $\mathrm{N}$, et al. Improvement of unilateral spatial neglect with numbering. Neurology 1990;40:1395-8. 